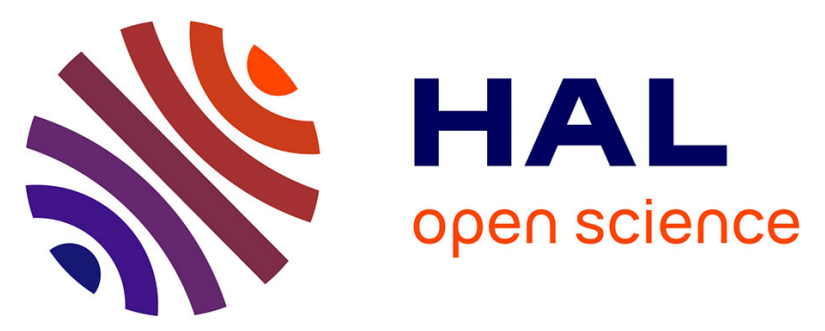

\title{
Understanding phase equilibria in high-entropy alloys: I. Chemical potentials in concentrated solid solutions - Atomic-scale investigation of AlCrFeMnMo \\ Rémy Besson
}

\section{- To cite this version: \\ Rémy Besson. Understanding phase equilibria in high-entropy alloys: I. Chemical potentials in con- centrated solid solutions - Atomic-scale investigation of AlCrFeMnMo. Journal of Alloys and Com- pounds, 2021, Journal of Alloys and Compounds, 872, pp.159745. 10.1016/j.jallcom.2021.159745 . hal-03256318}

\section{HAL Id: hal-03256318 \\ https://hal.univ-lille.fr/hal-03256318}

Submitted on 7 Oct 2021

HAL is a multi-disciplinary open access archive for the deposit and dissemination of scientific research documents, whether they are published or not. The documents may come from teaching and research institutions in France or abroad, or from public or private research centers.
L'archive ouverte pluridisciplinaire HAL, est destinée au dépôt et à la diffusion de documents scientifiques de niveau recherche, publiés ou non, émanant des établissements d'enseignement et de recherche français ou étrangers, des laboratoires publics ou privés. 


\title{
Understanding phase equilibria in high-entropy alloys: I. Chemical potentials in concentrated solid solutions - Atomic-scale investigation of AlCrFeMnMo
}

\author{
R. Besson \\ Univ. Lille, CNRS, INRAE, Centrale Lille, UMR 8207 - UMET - Unité Matériaux et Transformations, F-59000 Lille, France
}

\section{ART ICLE INFO}

\section{Article history:}

Received 11 February 2021

Received in revised form 20 March 2021

Accepted 26 March 2021

Available online xxx

\section{Keywords}

Density functional theory

Atomic-scale simulations

Multiprincipal metallic alloys

Chemical potentials

\begin{abstract}
A B S T R A C T
Atomic-scale simulations and modelling were carried out to investigate the thermodynamic properties of the bcc AlCrFeMnMo equiatomic high-entropy alloy (HEA) solid solution. Special care was taken to the determination of elemental chemical potentials, which are the central quantities governing the behaviour of HEAs in practical situations. The statistical properties of the disordered alloy were studied by extensive use of special quasirandom structures, including the influence of various types of short-range order. The local atomic order in AlCrFeMnMo appears to promote pairs of unlike atoms, although such non-random atomic arrangements are at odds with commonly admitted pictures for HEAs. While the chemical potentials derived from our moderate-size $(\sim 1000$ alloy configurations) statistical sample reveal an overall agreement on trends, more quantitative estimations of these quantities remain tricky. A significant $(\sim 0.5 \mathrm{eV})$ uncertainty should be taken into account when using chemical potentials in further studies involving HEA solid solutions in equilibrium with other phases.
\end{abstract}

(C) 2021

\section{Introduction}

Due to their promising properties, high-entropy alloys (HEAs) constitute a class of materials currently under many kinds of investigations, either experimental or theoretical [1]. More specifically, the AlCrFeMnMo system has been paid little attention up to now [2]. Nevertheless, recent experimental studies [3] have pointed out the interest of exploring this quinary system to elaborate body-centered cubic (bcc) single-phase disordered alloys offering good properties for coatings with various thicknesses between 0.1 and $100 \mu \mathrm{m}$. While achieving this purpose implies to optimize the composition of the desired bcc AlCrFeMnMo solid solution (s.s.), practical elaboration also requires to overcome many difficulties, related to the control of the phases, compositions and degrees of shortand long-range atomic order present in the alloy. In addition, ensuring stability for the target alloy implies to have a good knowledge of its thermodynamic properties. In this context, the chemical potentials of the various metallic elements involved in the s.s. are central quantities to determine. This issue becomes especially critical in presence of so-called "second-phase particles", since in such situations, the s.s. metallic chemical potentials, through the well-known rules of equilibria, have a direct influence on the properties of these particles. Even more critically, the multi-elemental nature of HEAs allows the simultaneous presence of a relatively large number of (un)desired additional non-s.s. phases, and controlling their stability, either to promote or to discard them, passes through a better knowledge of the metallic chemical potentials in the main s.s.
The aim of our work is to use atomic-scale simulations and modellings to investigate the thermodynamic features of bcc solid solutions of HEAs within the quinary AlCrFeMnMo system. To this aim, ab initio energy calculations and thermodynamic modellings of the metallic chemical potentials (section II) will be employed, to investigate the properties of the solid solution (section III), and allowing to discuss their implications for the elaboration of AlCrFeMnMo HEAs (section IV).

\section{Methods}

\subsection{Ab initio calculations}

Modelling of the bcc AlCrFeMnMo solid solution was achieved by means of special quasirandom structures (SQS [4], see next section for details about SQS selection) built in various 54-site supercells containing $3 \times 3 \times 3$ bcc unit cells, this moderate size being chosen to maintain a reasonable computational cost, due to the relatively high number of SQSs considered. The required ab initio calculations were performed with the VASP software [5], using the Projector Augmented Wave mode [6] and the GGA approximation with the PBE functional $[7,8]$. The energy cutoff for the plane wave expansion was chosen equal to $560 \mathrm{eV}$ throughout. The k-point mesh was of Monkhorst-Pack type [9], with $7 \times 7 \times 7$ grids for the solid solution. These cutoff and k-point values ensured reasonable accuracy for all total energies. All calculations were spin-polarized (collinear mode), and earlier studies [10] suggested to use zero initial magnetic moments for all species, except Fe atoms for which the usual value $\mu(\mathrm{Fe})=2.2 \mu_{\mathrm{B}}$ was specified. In all 
cases, the SQS optimization included atomic relaxations and supercell shape.

\subsection{Generation of SQSs with controlled short-range order for bcc quinary AlCrFeMnMo}

The SQS approach offers a convenient path to build specific alloy configurations that realistically mimic the fully disordered alloy (perfect random alloy, occurring in the high-temperature limit), i.e. the solid solution with no short-range order (s.r.o.). The SQS optimization was achieved by least-square minimization, via a Metropolis Monte-Carlo algorithm, of the usual (i.e. restricting to the first few short-ranged pairs) s.r.o. parameters.

$\alpha_{i j}^{n}$ on the first two neighbour shells, these quantities being defined, for each pair $\mathrm{i}<\mathrm{j}$ of chemical species and neighbour shell $\mathrm{n}$, as:

$\alpha_{i j}^{n}=1-\frac{p_{i j}^{n}}{x_{j}}$

where $p_{i j}^{n}$ is the probability that the neighbour shell $\mathrm{n}$ of species $\mathrm{i}$ is occupied by species $\mathrm{j}$, and $x_{j}$ is the atomic fraction of $\mathrm{j}$ in the s.s. Various SQSs could thus be obtained by including either $\left\{\alpha_{i j}^{1}\right\}_{i<j}$ only (first neighbour shell, noted $1 \mathrm{~N}$ below), or both $\left\{\alpha_{i j}^{1}\right\}_{i<j}$ and $\left\{\alpha_{i j}^{2}\right\}_{i<j}$ (first and second neighbour shells, $1 \mathrm{~N}$ and $2 \mathrm{~N}$ ), in the optimization search. Moreover, several distinct disordered configurations were conveniently produced, by considering the set of "chemical" permutations, i.e. all those permutations of the chemical species on the sites of a given SQS consistent with the requirement of keeping the same composition. This provided a convenient way of estimating the average energy for a given composition in the disordered s.s, directly related to the energy part of the chemical potentials. In addition, by increasing "naturally" the number of disordered configurations, this systematic procedure offered the advantage of yielding some rather fundamental statistical information on the energy density of states of AlCrFeMnMo, namely some insight into the energy dispersion (for given mole numbers) characteristic of the disordered s.s. This dispersion, which should theoretically vanish for a perfectly random alloy, thus offers a direct measurement of the departure of AlCrFeMnMo from perfect randomness. It should however be noted that the maximal number of iso-composition SQS samples thus produced depends on the permutation "symmetry" of the composition considered, i.e. 24 samples for $\mathrm{A}_{11} \mathrm{~B}_{11} \mathrm{C}_{11} \mathrm{D}_{11} \mathrm{E}_{10}$ obtained by (A,B,C,D) permutations.

Various kinds of target typical s.r.o. environments were considered for these SQSs:

$*$ all $\alpha_{i j}=0$ : this corresponds to the usual concept of SQS, with no short-range order, at least on the closest neighbour shells.

$*$ all $\alpha_{i j}=\alpha_{\min }$ : in this case, on a given neighbour shell, each atom of type $i$ is surrounded equiprobably by atoms of other types $j \neq i$. For a quinary system, the theoretical value is $\alpha_{\min }=-1 / 4$, which could be roughly achieved in practice, at least when including only one neighbour shell in the optimization. Reaching this target in presence of two neighbour shells was found more difficult.

* all $\alpha_{\mathrm{ij}}=\alpha_{\text {max }}$ : on a given neighbour shell, each atom of type $\mathrm{i}$ is surrounded only by atoms of same type i. Although the expected value is $\alpha_{\max }=1$, this value could not be reached in practice, due to the limited size $3 \times 3 \times 3$ of the SC, but only $\alpha_{\max }(3 \times 3 \times 3) \sim 0.3$ could be obtained. We verified this hypothesis by calculations on $5 \times 5 \times 5$ SC, which yielded higher values of $\alpha_{\max }$, for instance, $\alpha_{\max }(5 \times 5 \times 5) \sim$ 0.5 .

We stick to situations where all $\alpha_{i j}$ have the same value, so that this s.r.o. state is preserved when applying the permutations of chemical species to a given SQS. The approach proposed here, relying on the permutations of an SQS, by providing some significant statistics, offers a convenient way of probing the density of states of the alloy at a given composition. It allows (i) evaluating "constrained" chemical potentials for various kinds of s.r.o., (ii) estimating the total width and baseline of the constant-composition energy spectrum of the disordered nearly random alloy, a useful information as shown below.

\subsection{Chemical potentials from $S Q S s$}

The elemental chemical potentials in the s.s. were obtained from these SQS-based investigations, through the standard general expression:

$\mu_{j}=\frac{\partial G}{\partial N_{j}}$

with $\mathrm{G}=\mathrm{E}-\mathrm{TS}+\mathrm{PV}$ the s.s. free energy ( $\mathrm{P}=0$ here).

The identity $G\left(\left\{N_{i}\right\}, P, T\right)=N g\left(\left\{x_{i}\right\}_{i \neq i_{0}}, P, T\right)$ for any chemical species $\mathrm{i}_{0}$ (arbitrarily chosen as reference) yields:

$\mu_{j}=g+\sum_{i \neq i_{0}}\left(\delta_{i j}-x_{i}\right) \frac{\partial g}{\partial x_{i}}$

The main issue then amounts to finding an efficient use of this general relation in the context of the SQS modelling of HEAs. The panel of symmetry permutations available for each nearly equiatomic SQS of type $A_{11} B_{11} C_{11} D_{11} E_{10}$ provides an elementary way to evaluate the energy part $e$ of $g \hat{=} e-T s$, by introducing the temperature dependence via thermodynamic weights $e^{-\beta E_{\lambda}} / Z_{r}(\beta=1 / k T)$ associated with each 54-site SQS configuration $\lambda$ for a given composition $C=\left\{N_{i}\right\}$, and a partition function restricted to the subset (noted $\Pi[S Q S](C)$ ) of those permutations of the SQS having this composition:

$$
Z_{r}[S Q S](C)=\sum_{\lambda \in \Pi[S Q S](C)} e^{-\beta E_{\lambda}}
$$

This "restricted partition function" (RPF) framework allows determining an averaged composition- (and temperature-) dependent alloy energy $\bar{e}$, the derivatives $\left\{\frac{\partial \bar{e}}{\partial x_{i}}\right\}_{i \neq i_{0}}$ being then deduced from the five permutation-based $\mathrm{A}_{11} \mathrm{~B}_{11} \mathrm{C}_{11} \mathrm{D}_{11} \mathrm{E}_{10}$ symmetrical compositions. As usually for HEAs, we assume for the entropy contribution $s$ the ideal form:

$s=S / N=-k \sum_{i} x_{i} \ln x_{i}$

from which:

$\frac{\partial S}{\partial N_{j}}=s+\sum_{i \neq i_{0}}\left(\delta_{i j}-x_{i}\right) \frac{\partial s}{\partial x_{i}}$

The chemical potentials then take the form:

$\mu_{j}=\tilde{\mu}_{j}+k T \ln x_{j}$

with:

$\tilde{\mu}_{j}=\bar{e}+\sum_{i \neq i_{0}}\left(\delta_{i j}-x_{i}\right) \frac{\partial \bar{e}}{\partial x_{i}}$

While the focus of this work is on the evaluation of $\tilde{\mu}_{j}$ from ab initio calculations, it can be mentioned that the contribution of the configuration entropy $k T \ln x_{j}$ in the quinary equiatomic alloy is equal to $-0.107 \mathrm{eV}$ for all elements at $800 \mathrm{~K}$ (a typical temperature for elaboration processes of HEAs). While this moderate constant shift is immaterial for the present purpose, it should nevertheless be taken into account in further applications on thermodynamic equilibria with HEA solid solutions with second-phase particles, the relevant quantities then being the full chemical potentials $\mu_{\mathrm{j}}$ rather than the $\tilde{\mu}_{\mathrm{j}}$ parts. It should also be kept in mind that the configurational entropy of an alloy can be described us- 
ing Eq. (5a) only for an ideal random solid solution (case " $\alpha_{\mathrm{ij}}=0$ "). In alloys with s.r.o. (e.g. cases " $\alpha_{\mathrm{ij}}=\alpha_{\min }$ " or " $\alpha_{\mathrm{ij}}=\alpha_{\max }$ "), the configurational entropy is usually smaller [11].

This RPF approach to get the chemical potentials in an HEA solid solution, while simple and convenient, however suffers from some arbitrariness, due to the choice of the SQS used as input, an issue that will be illustrated in the next section. To remedy this deficiency, a simple model, labelled "power DOS" (PDOS) below, was also introduced, using for the configurational degrees of freedom of the alloy a density of states of the form $g(E)=A\left(E-E_{0}\right)^{N}$, with $N$ the number of atoms and $E_{0}$ the bottom ("ground state") of the iso-composition energy spectrum for this $\mathrm{N}$-atom system, both $E_{0}$ and the normalization constant A depending on the composition, and $g(E)$ being zero for $\mathrm{E}>\mathrm{E}_{0}+\Delta \mathrm{E}$ (with $\Delta \mathrm{E}$ the width of the density of states for this $\mathrm{N}$-atom system). Such a general form is found to be reasonable from general arguments of statistical physics [12]. In this alternative approach, the whole information available in the full set of SQSs may be used to determine the parameters $A\left(\left\{x_{i}\right\}\right)$ and $E_{0}\left(\left\{x_{i}\right\}\right)$, thus in principle removing the RPF ambiguity due to the SQS choice. It is then straightforward to show (see Appendix 1 ) that the expression for the alloy chemical potentials in this context becomes:

$\tilde{\mu}_{j}=e_{0}+\sum_{i \neq i_{0}}\left(\delta_{i j}-x_{i}\right) \frac{\partial e_{0}}{\partial x_{i}}+\mu_{j}^{X}$

with the "excess" term:

$\mu_{j}^{X}=k T\left[1+\ln \left(\frac{\Delta \mathrm{e}}{k T}\right)+\frac{1}{\Delta \mathrm{e}} \sum_{i \neq i_{0}}\left(\delta_{i j}-x_{i}\right) \frac{\partial \Delta \mathrm{e}}{\partial x_{i}}\right]$

The energies per atom $e_{0}\left(\left\{x_{i}\right\}\right)=E_{0} / N$ and $\Delta e\left(\left\{x_{i}\right\}\right)=\Delta E / N$ depend on the composition, and the link between $e_{0}$ and $\bar{e}$ defined previously is $e_{0}=\bar{e}(T \rightarrow 0 K)$. It should be noted that, contrary to the previous approach (eq. (5)), the ideal form of the configurational entropy in Eq. (6) is no longer an assumption, but derives naturally in PDOS from the shape chosen for $g(E)$.

\section{Results}

\subsection{SQSs for the nearly equiatomic bcc AlCrFeMnMo solid solution}

Following the lines described in the previous section for SQS generation, we thus started by producing a panel of six 54-site SQS configurations for a nearly equiatomic quinary bcc alloy, the optimized s.r.o. parameters of which are reported in Table 1 . As expected, confining to
$1 \mathrm{~N}$ optimization yielded rather homogeneous s.r.o. spectra, while this homogeneity was somewhat reduced when including the $2 \mathrm{~N}$ shell. Nevertheless, referring to probabilities on neighbour shells (Eq. (1)), the values indeed demonstrate three qualitatively distinct behaviours. This reflects clearly distinct types of chemical environments in the alloy, providing a useful basis for statistical and thermodynamic analysis. Fig. 1.

As outlined above, for each of these six SQSs, applying (A,B,C,D) permutations for fixed $\mathrm{E}$ allows to get a 24-element sample, and varying $\mathrm{E}(=\mathrm{Al}, \mathrm{Cr}, \mathrm{Fe}, \mathrm{Mn}, \mathrm{Mo}$ ) allows to explore five symmetrical nearly equiatomic compositions, each of which is described by a panel of 144 configurations. The statistical distributions of iso-composition total energies per atom for these SQS populations are displayed in Fig. 2 in the form of histograms, where each colour refers to a type of s.r.o., while full-colour and hatched parts refer to $1 \mathrm{~N}$ and $1 \mathrm{~N} 2 \mathrm{~N}$ optimizations respectively. The influence of the s.r.o. state is clearly visible, being responsible for the presence of several modes in the statistical distribution at each composition. The overall bell-shape of these energy spectra gives good confidence that the main features (centre and width) of the iso-composition DOS of AlCrFeMnMo are captured in the samples considered. It suggests that choosing a DOS width $\sim 100 \mathrm{meV} /$ atom should be reasonable in the nearly equiatomic composition range.

The influence of the alloy composition, for a given state of s.r.o., on the energy spectra of these SQS is represented in Fig. 2. It shows that switching from $\alpha_{\mathrm{ij}}=0$ to $\alpha_{\mathrm{ij}}=\alpha_{\min }$ implies for the current alloy energetics an overall downward shift of $\sim 25 \mathrm{meV} /$ atom. Conversely, the width of the DOS $\left(\alpha_{\mathrm{ij}}\right)$ is moderately affected. However, it should be noted that the total width of the DOS for the s.s. should be obtained by superimposing the partial DOSs for given $\alpha_{i j}$. These results point out a significant influence of s.r.o. on the energetics of the bcc AlCrFeMnMo solid solution. In particular, at least when only the first two neighbour shells are considered, the random solution appears not to be the most stable state of the alloy, especially when compared to the case $\alpha_{i j}=\alpha_{\text {min }}$ which corresponds to the situation where each atom is surrounded, with equal probabilities, by atoms of other types.

As another feature expected from AlCrFeMnMo bcc solid solutions, the width of each spectrum appears to be rather moderate and insensitive to the s.r.o. state of the alloy, being lower than $\sim 100 \mathrm{meV} /$ atom in all cases. The quantitative validity of this conclusion may of course be questioned, due to the limited SQS statistics. However, this probably evidences a clear-cut and reliable qualitative trend of the energetics in the quinary system considered. Noticeably, the iso-composition energy dispersion of the s.s., as deduced from our SQS calculations, offers the

\section{Table 1}

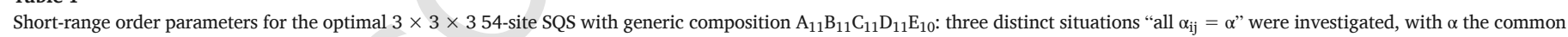

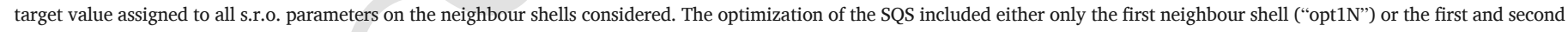
ones ("opt1N2N").

\begin{tabular}{|c|c|c|c|c|c|c|c|c|c|}
\hline & \multirow{2}{*}{$\begin{array}{l}\text { all } \alpha_{\mathrm{ij}}=0 \\
\text { opt1N }\end{array}$} & & & \multicolumn{3}{|c|}{ all $\alpha_{\mathrm{ij}}=\alpha_{\min }$} & \multicolumn{3}{|c|}{ all $\alpha_{\mathrm{ij}}=\alpha_{\max }$} \\
\hline & & opt1N2N & & opt1N & opt1N2N & & opt1N & opt1N2 & \\
\hline & $1 \mathrm{~N}$ & $1 \mathrm{~N}$ & $2 \mathrm{~N}$ & $1 \mathrm{~N}$ & $1 \mathrm{~N}$ & $2 \mathrm{~N}$ & $1 \mathrm{~N}$ & $1 \mathrm{~N}$ & $2 \mathrm{~N}$ \\
\hline$\alpha_{A B}$ & -0.004 & -0.004 & -0.041 & -0.227 & -0.116 & -0.190 & 0.275 & 0.219 & 0.256 \\
\hline$\alpha_{A C}$ & -0.004 & 0.052 & -0.041 & -0.227 & -0.116 & -0.190 & 0.275 & 0.107 & 0.256 \\
\hline$\alpha_{\mathrm{AD}}$ & -0.004 & -0.004 & 0.033 & -0.227 & -0.171 & -0.190 & 0.275 & 0.107 & 0.405 \\
\hline$\alpha_{\mathrm{AE}}$ & 0.018 & 0.080 & -0.064 & -0.227 & -0.166 & -0.145 & 0.325 & 0.386 & 0.182 \\
\hline$\alpha_{\mathrm{BC}}$ & -0.004 & -0.004 & -0.041 & -0.227 & -0.171 & -0.190 & 0.275 & 0.331 & 0.405 \\
\hline$\alpha_{\mathrm{BD}}$ & -0.004 & -0.004 & -0.041 & -0.227 & -0.116 & -0.190 & 0.331 & 0.442 & 0.256 \\
\hline$\alpha_{\mathrm{BE}}$ & 0.018 & 0.018 & 0.018 & -0.227 & -0.166 & -0.145 & 0.264 & 0.264 & 0.345 \\
\hline$\alpha_{\mathrm{CD}}$ & -0.004 & 0.052 & 0.033 & -0.227 & -0.116 & -0.116 & 0.275 & 0.554 & 0.405 \\
\hline$\alpha_{\mathrm{CE}}$ & 0.018 & 0.018 & -0.064 & -0.227 & -0.166 & -0.227 & 0.325 & 0.264 & 0.182 \\
\hline$\alpha_{\mathrm{DE}}$ & 0.018 & 0.080 & 0.018 & -0.227 & -0.166 & -0.227 & 0.264 & 0.141 & 0.182 \\
\hline
\end{tabular}




\section{$\alpha_{\mathrm{ij}}=\alpha_{\min } 0 \alpha_{\max }$ \\ Opt1N Opt1N2N \& 88}

Ceord. = 1 - AllICrIIFe10Mal1Moll
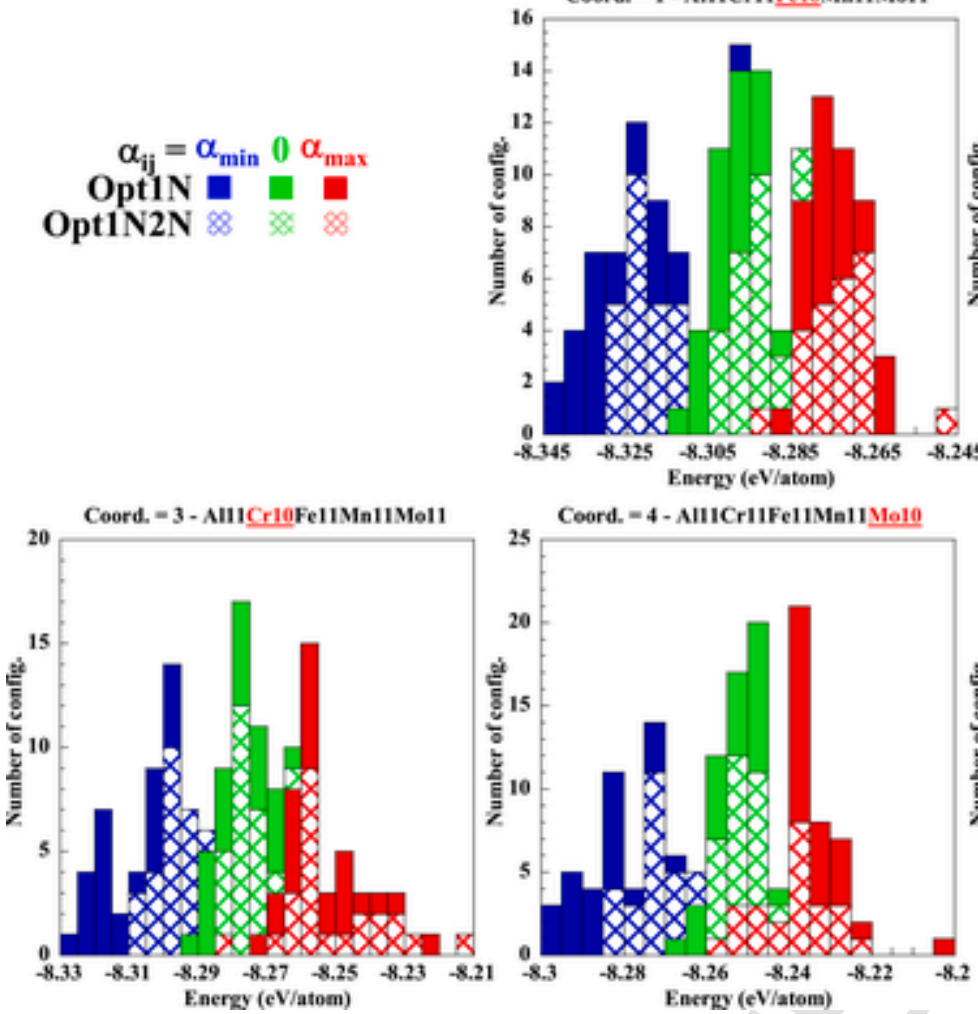

Coord. = 4-AlliCrilfe11Mal1 Malo

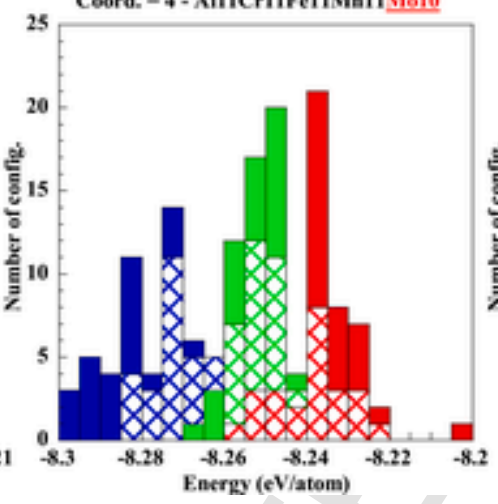

Coord. = 2 - AlIICrIIFelIMnI0MolI

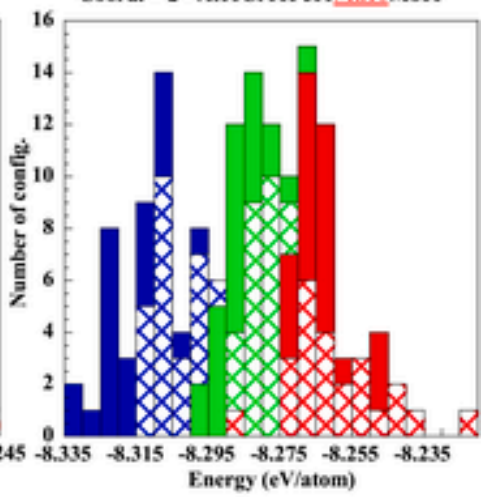

Coord, $=5$ - Al10Cr11FelIMnIIMe11

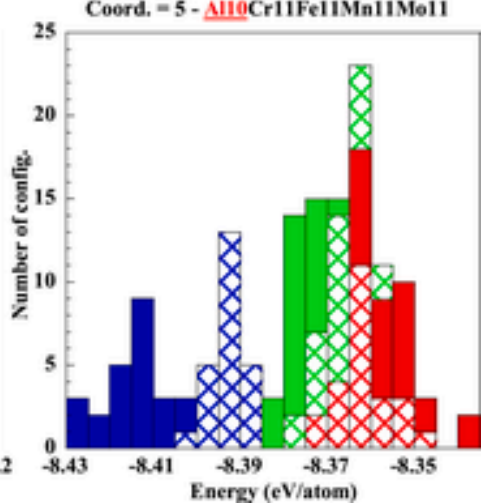

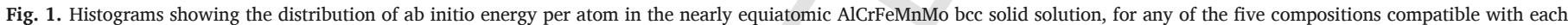

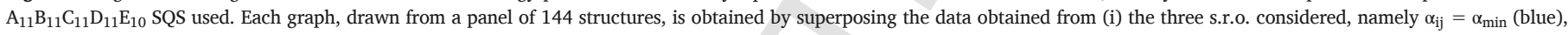
$\alpha_{i j}=0$ (green), $\alpha_{i j}=\alpha_{\max }$ (red), and (ii) the two SQS optimization frames (either opt1N or opt1N2N in fully coloured and hatched parts respectively).

\section{Opt1N口 Opt1N2N}

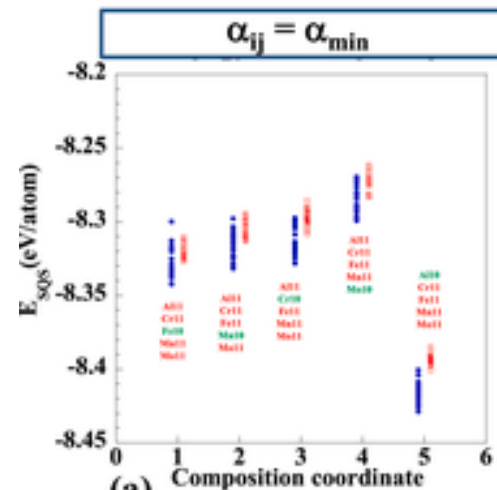

(a)

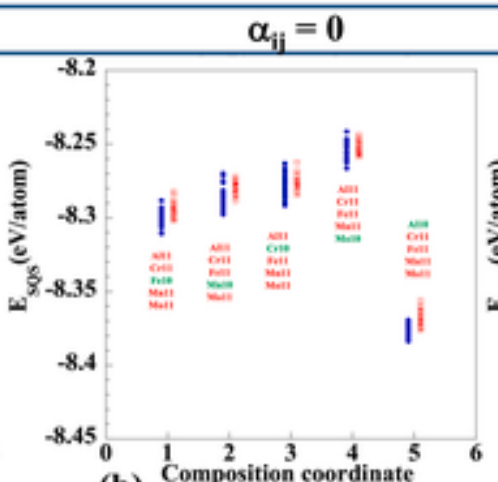

(b)

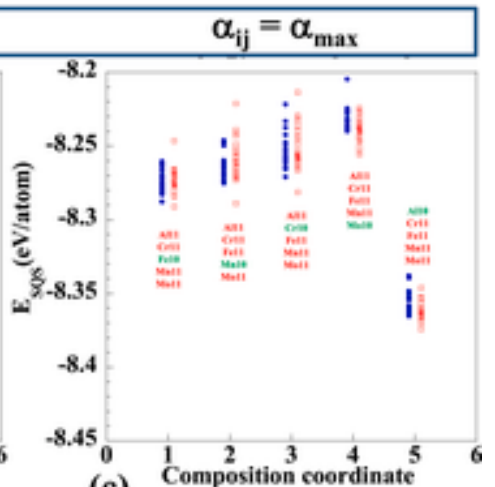

(c)

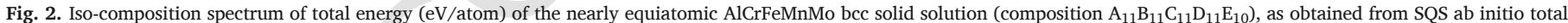

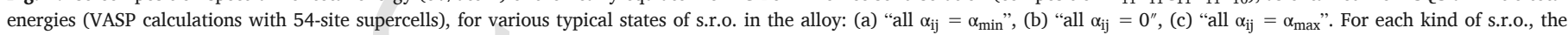
influence of including one (full symbols) or two (open symbols) neighbour shells in SQS optimizations is also displayed.

advantage of providing an insight into the degree of perfect randomness of the alloy at any temperature of interest. Using the well-known equivalence $25 \mathrm{meV} \equiv 300 \mathrm{~K}$, this energy dispersion of SQSs with various s.r.o. strongly suggests that temperatures around $1000 \mathrm{~K}$, a practically reasonable range in elaboration processes, should be sufficient for the $\mathrm{AlCrFeMnMo}$ bcc s.s. to reach a reasonable degree of perfect randomness. However, this overall trend should also depend on the possibility of partial long-range ordering (LRO) in each composition domain: the determination of the width of the energy spectrum for such LRO states, as well as its absolute level compared to the s.s., constitutes an important issue that should be investigated further. As for the present work, these results give reasonable confidence in the accuracy of the subsequent investigations of chemical potentials in AlCrFeMnMo solid solutions.

\subsection{Chemical potentials in the bcc AlCrFeMnMo solid solution}

Using the above SQS energetics, the chemical potentials in the bcc AlCrFeMnMo equiatomic solid solution were calculated. The available panel of ab initio data allowed examining in detail the effect of the parameters selected (s.r.o. and range of optimization) when building the SQSs. More precisely, in a first step, the chemical potentials were determined, both in the RPF and PDOS approaches, by using as input data the energetics of a given SQS and its permutations. The corresponding 
results at $800 \mathrm{~K}$ are displayed in Tables $2-3$ and Fig. 3. In the case of RPF (Table 2 and Fig. 3a), the chemical potentials are found to be sensitive to the degree of s.r.o, with variations ranging between 0.3 and $0.5 \mathrm{eV}$ for all elements. The effect of the optimization scheme is also important, with a dispersion that may reach $0.5 \mathrm{eV}$, being somewhat lower for $\alpha_{i j}=0$. Using the PDOS approach (Table 3 and Fig. 3b), the results are significantly different from RPF, with a strong sensitivity to the SQS selected, and a large dispersion $\sim 2 \mathrm{eV}$ for all elements. This emphasizes the importance, in the PDOS framework, of estimating as accurately as possible the baseline and width of the alloy DOS. This approach may be made tricky by the presence of isolated points on both

Table 2

Chemical potentials $\widetilde{\mu}(\mathrm{eV})$ in the bcc AlCrFeMnMo solid solution around the equiatomic composition domain, deduced from SQS calculations and the "restricted partition function" approach, using as input the sets of SQSs built for various target values of s.r.o. parameters $\alpha_{i j}$ and ranges $(1 \mathrm{~N}$ or $1 \mathrm{~N} 2 \mathrm{~N}$ ) of optimization for these parameters (as listed in Table 1). These chemical potentials are given at $800 \mathrm{~K}$, the effect of temperature appearing through the thermodynamic weight of each SQS (see text). The influence of temperature, as taken into account from equation (5), is found to be negligible between 0 and $800 \mathrm{~K}$.

\begin{tabular}{lllllll}
\hline \multirow{2}{*}{$\mathrm{RPF}-5 \times 24$ config. } & & $\tilde{\mu}(\mathrm{Al})$ & $\tilde{\mu}(\mathrm{Cr})$ & $\tilde{\mu}(\mathrm{Fe})$ & $\begin{array}{l}\tilde{\mu} \\
(\mathrm{Mn})\end{array}$ & $\tilde{\mu}(\mathrm{Mo})$ \\
\hline \multirow{2}{*}{$\alpha_{\mathrm{ij}}=0$} & Opt1N & -4.350 & -9.304 & -8.260 & -8.976 & -10.655 \\
\multirow{2}{*}{$\alpha_{\mathrm{ij}}=\alpha_{\min }$} & Opt1N2N & -4.301 & -9.239 & -8.297 & -9.009 & -10.657 \\
& Opt1N & -3.886 & -9.306 & -8.536 & -9.123 & -10.878 \\
$\alpha_{\mathrm{ij}}=\alpha_{\max }$ & Opt1N2N & -4.311 & -9.306 & -8.311 & -9.062 & -10.637 \\
& Opt1N & -4.132 & -9.171 & -8.280 & -8.973 & -10.879 \\
& Opt1N2N & -4.205 & -9.211 & -8.674 & -8.789 & -10.612 \\
\hline
\end{tabular}

Table 3

Chemical potentials $\tilde{\mu}(\mathrm{eV})$ in the bcc AlCrFeMnMo solid solution around the equiatomic composition domain, within the "power DOS" approach (same details as in Table 2).

\begin{tabular}{lllllll}
\hline PDOS - $5 \times 24$ config. & $\tilde{\mu}(\mathrm{Al})$ & $\tilde{\mu}(\mathrm{Cr})$ & $\tilde{\mu}(\mathrm{Fe})$ & $\tilde{\mu}(\mathrm{Mn})$ & $\tilde{\mu}(\mathrm{Mo})$ \\
\hline \multirow{2}{*}{$\alpha_{\mathrm{ij}}=0$} & Opt1N & -2.804 & -10.021 & -8.021 & -9.607 & -10.778 \\
\multirow{3}{*}{$\alpha_{\mathrm{ij}}=\alpha_{\min }$} & Opt1N2N & -4.572 & -9.923 & -8.373 & -8.556 & -9.854 \\
& Opt1N & -3.269 & -8.948 & -9.530 & -9.122 & -10.426 \\
$\alpha_{\mathrm{ij}}=\alpha_{\max }$ & Opt1N2N & -3.573 & -9.939 & -7.827 & -8.912 & -11.141 \\
& Opt1N & -3.318 & -10.845 & -7.480 & -8.366 & -10.987 \\
& Opt1N2N & -2.535 & -10.635 & -8.338 & -10.217 & -9.202 \\
\hline
\end{tabular}

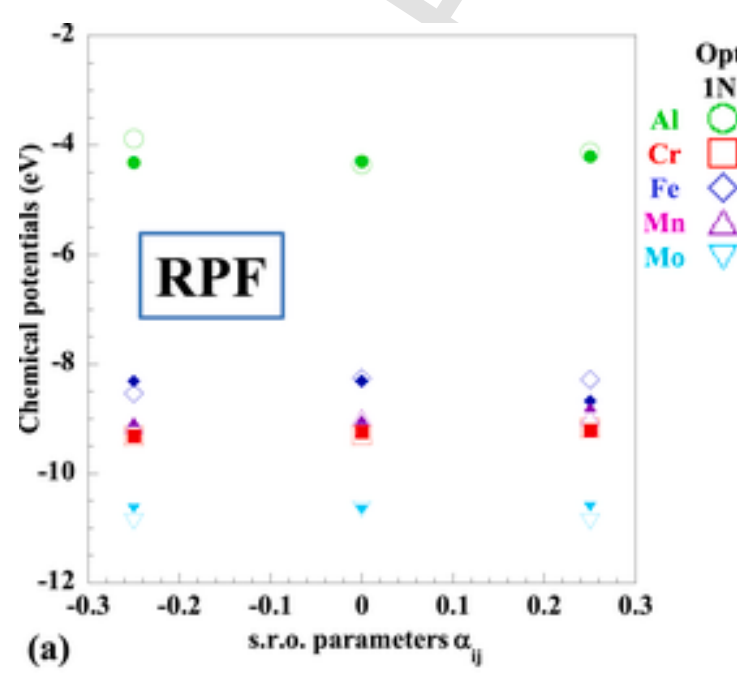

extremities of the iso-composition structure set (see Fig. 2), which may be responsible for biasing the power law approximation of the DOS.

While the above results have clearly illustrated the inherent difficulties when attempting to use SQSs to evaluate chemical potentials in HEAs, it should be noted that there is no reason for privileging a priori a given type of s.r.o. in the SQSs selected, and the same remark holds for the optimization range, the role of which was also shown not to be negligible. Therefore, it is logical to revisit both the RPF and PDOS approaches, using the full statistics allowed by the 720-element SQS panel available. The parameters required for PDOS in this context are reported in Table 4. The compositional derivatives of the DOS width and the excess part of the chemical potentials are also displayed (first and second rows of Table 5), in order to show that these quantities are not negligible. The last row of Table 5 provides the energies of the pure elements in bcc structure, since contrary to chemical potentials, these quantities are easy to obtain. The overall chemical potentials are reported in Table 5 and displayed in Fig. 4. In the RPF approach, the results are identical as those obtained previously (third row of Table 2), as expected from the larger stability (Fig. 2) of SQS configurations obtained in "all $\alpha_{i j}=\alpha_{\min }$ " and "Opt1N" conditions. As for PDOS, the use of the 720 -element set allows to recover chemical potentials in closer agreement with their RPF counterparts. Nevertheless, confronting RPF and PDOS shows that, even using this more extended statistical sample, the elemental chemical potentials in the AlCrFeMnMo solid solution should carry a significant dispersion of $\sim 0.5 \mathrm{eV}$. It may be noticed that the chemical potential fluctuations seem more important for $\mathrm{Al}$ and Mo than for the other elements considered. Interestingly, bcc elemental energies appear as a rather reasonable approximation for the chemical potentials in a disordered equiatomic environment, though it would be interesting to check its validity in other complex concentrated alloys.

\section{Discussion}

The present work, devoted to the estimation, from SQS-based atomic-scale calculations, of the chemical potentials in concentrated multi-element alloys such as HEAs, reveals interesting features concerning the energetics of these rather complex chemical environments. Noticeably, our results provide a reasonable idea of the inherent accuracy, found to be typically $0.5 \mathrm{eV}$, associated with the chemical potentials in HEA-type solid solutions obtained from moderately large statistical ( 1000-element) samples. This typical "error bar", which seems difficult to reduce, should be taken into account when carrying out further investigations, especially on the various equilibria possible between a

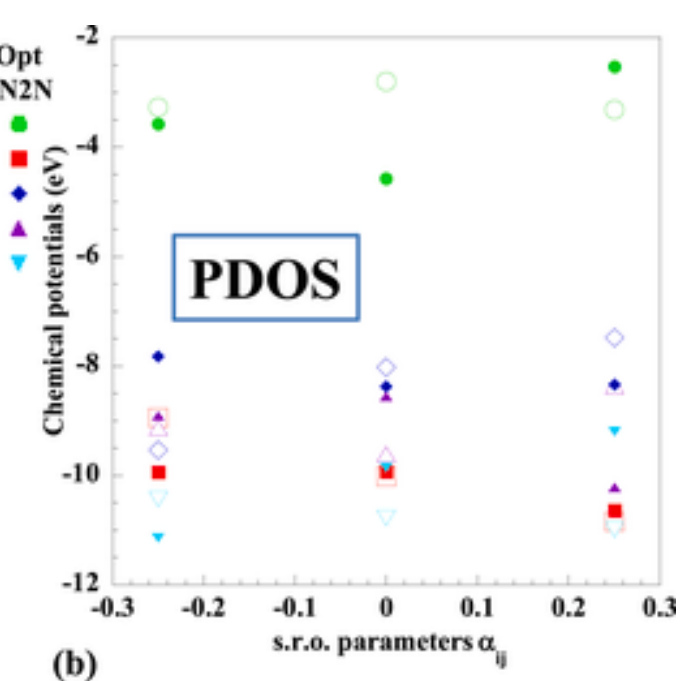

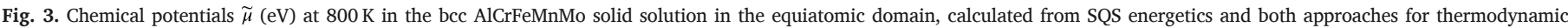

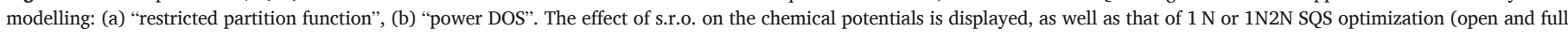
symbols respectively, same meaning as Fig. 2). 
Table 4

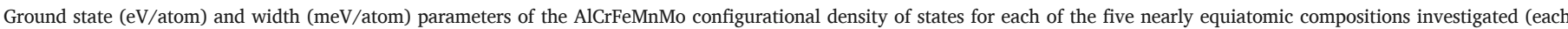

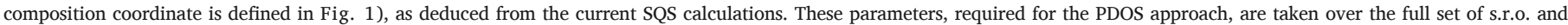
optimization schemes, i.e. over a 720-element panel of 144 SQSs for each composition.

\begin{tabular}{|c|c|c|c|c|c|c|c|c|c|}
\hline Coord. $=1$ & & Coord. $=2$ & & Coord. $=3$ & & Coord. $=4$ & & Coord. $=5$ & \\
\hline$e_{0}$ & $\Delta \mathrm{e}$ & $\mathrm{e}_{0}$ & $\Delta \mathrm{e}$ & $\mathrm{e}_{0}$ & $\Delta \mathrm{e}$ & $\mathrm{e}_{0}$ & $\Delta \mathrm{e}$ & $\mathrm{e}_{0}$ & $\Delta \mathrm{e}$ \\
\hline-8.343 & 97 & -8.332 & 111 & -8.328 & 114 & -8.299 & 95 & -8.429 & 83 \\
\hline
\end{tabular}

Table 5

DOS derivatives (eV/atom), excess terms (eV) and elemental chemical potentials $\widetilde{\mu}(\mathrm{eV})$ in the AlCrFeMnMo equiatomic solid solution, calculated at $800 \mathrm{~K}$ from SQS calculations. The entry for the Mo derivative is empty, because $\mathrm{x}_{\mathrm{Mo}}$ was taken as the dependent atomic fraction. The chemical potentials are given either in the RPF (third row, eq. (5)) or in the PDOS approach (fourth row, Eq. (6)). All the quantities in the first four rows were obtained from the full panel of $5 \times 144=720$ configurations deduced from the permutations of the six SQSs considered. The last row provides the energies of the pure elements in bcc structure for purpose of comparison.

\begin{tabular}{llllll}
\hline $5 \times 144$ config. & $\mathrm{Al}$ & $\mathrm{Cr}$ & $\mathrm{Fe}$ & $\mathrm{Mn}$ & Mo \\
\hline$\frac{\partial \Delta \mathrm{e}}{\partial x_{i}}$ & 0.214 & -1.059 & -0.072 & -0.834 & - \\
$\mu^{\mathrm{X}}$ & 0.547 & -0.317 & 0.354 & -0.165 & 0.402 \\
$\tilde{\mu}^{R P F}$ & -3.886 & -9.306 & -8.536 & -9.123 & -10.878 \\
$\tilde{\mu}^{\text {PDOS }}$ & -3.336 & -9.631 & -8.185 & -9.284 & -10.472 \\
$E^{\mathrm{bcc}}$ & -3.648 & -9.506 & -8.306 & -8.877 & -10.944 \\
\hline
\end{tabular}

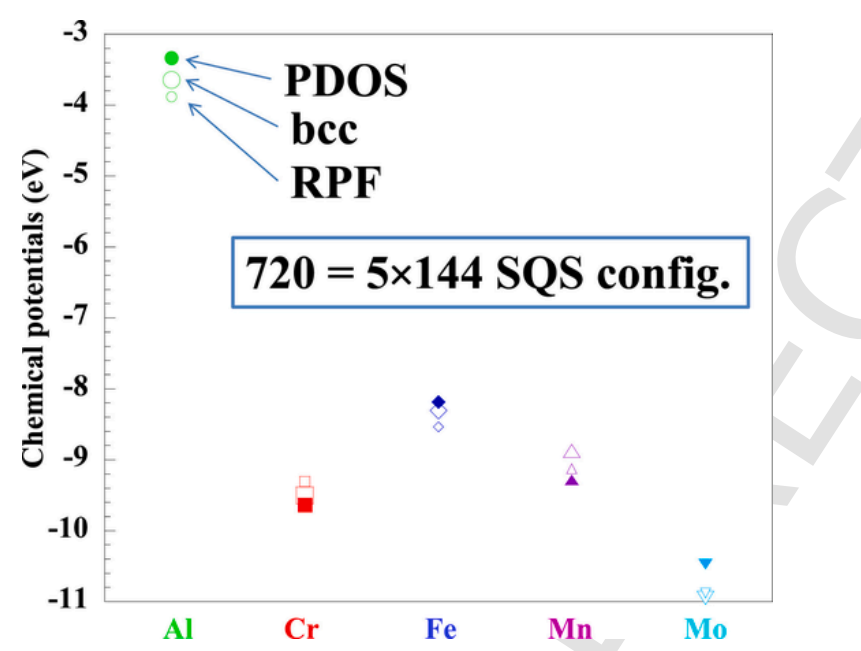

Fig. 4. Chemical potentials $\tilde{\mu}(\mathrm{eV})$ at $800 \mathrm{~K}$ in the bcc AlCrFeMnMo solid solution in the equiatomic domain, calculated from SQS energetics and thermodynamic modelling (cf. Table 5). Full symbols, small open symbols and large open symbols refer respectively to PDOS, RPF, using the full 720-element data set in both cases, and bcc elemental energies.

main HEA solid solution and e.g. second-phase particles or microstructural defects such as grain boundaries, since in all cases the chemical potentials in the solid solution are the central quantities governing the response of surrounding microstructures (at least if the alloy is close enough to equilibrium, which depends on the treatment imposed to it), as evidenced e.g. by atomic-scale modelling of ordered compounds [13]. Our work also demonstrates the importance of properly selecting the SQSs retained for the chemical potential investigations: it seems hazardous to select SQSs without any control of the internal variables, in particular the values and range of control of the short-range order parameters, characterizing these model random structures. Noticeably, by comparison of various typical states of s.r.o., our study clearly points out the irrelevance of selecting a priori the most disordered case ("all $\alpha_{i j}=0^{\prime \prime}$ ) as representative of the HEA. This conclusion is in agreement with previous recent studies [14] indicating that s.r.o parameters in HEAs should be positive for some pairs of species and negative for others. In this respect, while the AlCrFeMnMo alloy energy was found monotonic with s.r.o. (when restricting to a common value $\alpha_{i j}=\alpha$ ), it may be questioned whether the aforestudied cases "all $\alpha_{\mathrm{ij}}=\alpha_{\min }$ " and "all $\alpha_{\mathrm{ij}}=\alpha_{\max }$ " are indeed relevant, i.e. whether they are sufficient to provide a good picture of the total width of the alloy DOS. However, while further check of whether the two situations "all $\alpha_{\mathrm{ij}}=\alpha_{\min }$ " and "all $\alpha_{\mathrm{ij}}$ $=\alpha_{\max }$ " really correspond to global extrema of the alloy energetics in the equiatomic composition domain would doubtlessly be useful, this would require varying independently each of the ten s.r.o. parameters between $\alpha_{\min }$ and $\alpha_{\max }$, hence for a quinary system a set of $2^{10} \sim 1000$ states of s.r.o. to be explored, which seems totally out of reach of current computational facilities. High-quality experiments (e.g. on large instruments) providing reliable measurements of s.r.o. would be valuable in helping orientate the choice of SQSs with relevant s.r.o., but for the time being this still remains a hardly achievable task.

When performing ab initio-based studies of concentrated alloys containing magnetic elements such as $\mathrm{Fe}, \mathrm{Mn}$ or $\mathrm{Cr}$, another important issue that should be kept in mind concerns the possible role of the magnetic moments in the energetics of the HEA solid solution. It is a well-known fact that handling magnetic degrees of freedom (as done e.g. in spin-dependent SGGA calculations) significantly increases the difficulty, because it may introduce many local minima in the alloy energetics, making it much more tricky to determine the ground-state energy of each chemical configuration. More precisely, the energy obtained in practice might become strongly dependent on the particular values of the initial magnetic moments chosen in the simulations: for specific choices of these quantities, the system might remain trapped in a metastable magnetic state, leading to an overestimation of the energy for the alloy configuration. In our case, our assumption (initial magnetic moments on $\mathrm{Fe}$ atoms taken as $\mu(\mathrm{Fe})=2.2 \mu_{\mathrm{B}}$, initial values for all the other species chosen equal to zero) was found satisfactory for steels with a high concentration of Fe (as in Ref. [10]) but it may not obviously work for AlCrFeMnMo, where the concentration of $\mathrm{Fe}$ is only $20 \%$. To provide some insight into this issue, it may be useful to perform some inspection of the final magnetic moments, obtained after energy optimization of each SQS structure used in our work. This information is collected in Fig. A4 (Appendix 3), which displays the species-dependent magnetic moments on the 54 sites for the 120 permutations of each of the six SQS structures (one graph for each SQS). This emphasizes several species-dependent clear trends: (i) a roughly ferromagnetic state for $\mathrm{Fe}$ atoms, however somewhat attenuated (presence of antiparallel Fe spins) in the "all $\alpha_{\mathrm{ij}}=\alpha_{\min }$ " and "all $\alpha_{\mathrm{ij}}=\alpha_{\max }$ " cases; (ii) a roughly ferromagnetic coupling between Fe and Mn moments, though with a non-negligible anti-parallel component; (iii) a more "paramagnetic" behaviour for $\mathrm{Cr}$, which thus seems to have no evident magnetic coupling with Fe; (iv) a roughly (resp. strictly) non-magnetic behaviour for Mo and Al. These clear and seemingly consistent trends suggest that simulation artifacts (magnetic trapping) may be limited, giving hence reasonable confidence in the accuracy of our results on AlCrFeMnMo. In addition, we performed an analysis (limited to a single permutation of the (opt1N; $\left.\alpha_{i j}=0\right)$ SQS) of the effect of 
varying the $\mathrm{Cr}$ and $\mathrm{Mn}$ initial magnetic moments $(\mu(\mathrm{Cr} / \mathrm{Mn})=+/-1$ $\mu_{B}$ ) on the configuration energy: this confirmed that the potential energy gain should remain non-significant (below $1 \mathrm{meV} /$ atom). On the whole, investigating more thoroughly magnetism-related issues in HEA energetics would be relevant, but such an interesting task goes far beyond the scope of the present work.

Keeping in mind these limitations of SQS-based approaches, it would be interesting to explore their connections with alternative routes for thermodynamic calculations on HEAs, noticeably those relying on cluster expansions, since the latter have proved efficient in providing shortand long-range order as well as compositions and chemical potentials in alloys with significant complexity [10]. To this aim, the critical issue is to elaborate a reliable energy model for the alloy in the relevant composition range, which can hardly be carried out by conventional procedures of fitting of the model on ab initio input data. Therefore, it would be interesting to investigate the efficiency of alternative routes, also relying on ab initio data but free of any tricky fitting step, which have been proposed recently to obtain the required energy interactions $[15,16]$. An interesting feature of chemical potentials in AlCrFeMnMo, which could be tested by confronting to other approaches, concerns their relative proximity with the corresponding elemental bcc energies. The validity of this atomic-scale result, as well as the degree this can be considered as a general feature independent of the chemical elements, could be interestingly investigated by such cluster expansion modelling, as well as by more macroscopic, phenomenological, (e.g. Calphad-type) thermodynamic descriptions. While the absolute values of chemical potentials, because depending on arbitrary energy references, are meaningless in themselves, the connections between equiatomic chemical potentials and bcc elemental energies may be helpful, more generally in the field of HEAs, in providing a sound basis for comparing various types of modellings at different scales.

Using SQSs to probe HEAs implicitly relies on the hypothesis that the alloy energetics, at working temperatures, is not influenced by states with long-range order. The validity of this hypothesis depends on the energy levels of these long-range ordered states, which may a priori have some influence on the overall alloy chemical potentials. Extending the above considerations, the use of SQSs could be considered to investigate partial long-range order (starting with the simplest one $=\mathrm{B} 2$ ) in the same equiatomic quinary domain of AlCrFeMnMo. This would provide a more comprehensive picture of the alloy energetics in the equiatomic domain, in particular via the identification of possible long-range order showing some overlapping with the disordered (i.e. s.r.o.-based) alloy DOS.

The present work was exclusively performed with 54-site SQSs ( $3 \times 3 \times 3$ bcc unit cells), because this seemed to be a reasonable compromise between efficiency and limitation of spurious order due to the periodic boundary conditions. In particular, we were dissuaded by the latter requirement from employing smaller 16 -site SQSs $(2 \times 2 \times 2$ unit cells). Of course, extending the approach to larger systems (e.g. 128-site $4 \times 4 \times 4$ SQS) would provide valuable further insight in the properties of the disordered alloy, but due to its computational cost, this task was left for later works.

Finally, it may be useful to note that increasing computational power may soon help including other effects, beyond the configurational ones, potentially playing some role in HEA energetics. In particular, the effect of phonons on the DOS features should be easily, at least in principle, manageable in the above SQS-based framework. Analogies with earlier works on ordered alloys $[17,18]$ might suggest that phonons could entail some smoothing of the alloy energetics, possibly helping reduce the critical dispersion noted, hence allowing a more accurate determination of mostly important thermodynamic quantities such as HEA chemical potentials.

\section{Conclusion}

In this work, we used ab initio-based simulations and modelling to investigate the properties of the bcc equiatomic AlCrFeMnMo solid solution. We paid particular attention to the elemental chemical potentials, since these quantities are essential in order to interpret the behaviour of high-entropy alloys (HEA) often involved in multiphase equilibria. The inherently statistical issues related to the energetics of such concentrated disordered systems were taken into account by means of extensive application of special quasirandom structures (SQSs), in which various internal variables (kinds and ranges of short-range order) were monitored, leading to $\sim 10^{3}$ alloy configurations in the nearly equiatomic composition range. This SQS-based exploration depicted the energetics of the equiatomic alloy as an increasing function of the short-range order state. This marked preference, for each species, to be surrounded by other chemical types, may discard the usually admitted picture of AlCrFeMnMo HEAs as purely random alloys. For the thermodynamic modelling, we compared two approaches, relying either on restricted partition functions or on a model power law for the alloy density of states. We demonstrated that the step of SQS selection is critical, since the SQS internal variables have a critical influence on the resulting chemical potentials. While improved somewhat by extending the sample size, this issue could not be fully solved: our moderately large panel of configurations led to $\mathrm{a} \sim 0.5 \mathrm{eV}$ residual dispersion on the chemical potentials, a significant value that should be kept in mind when attempting further HEA studies in which these central quantities are required, e.g. when investigating microstructural defects or equilibria with second-phase particles.

\section{Declaration of Competing Interest}

The author declare that he has no known competing financial interests or personal relationships that could have appeared to influence the work reported in this paper.

\section{Acknowledgments}

The author warmly thanks the Centre de Ressources Informatiques (CRI) of the Université de Sciences et Technologies de Lille for computational facilities and technical support.

\section{Appendix 1. : Some details on the PDOS model}

We consider for the alloy density of states a "power law" model, i.e. $g(E)=A\left(E-E_{0}\right)^{N}$, between $E_{0}$ and $E_{1}=E_{0}+\Delta E$, with $E_{0}$ and $\Delta E$ respectively the bottom and width of the iso-composition energy spectrum for this $\mathrm{N}$-atom system. $g(E)$ is supposed to vanish for $\mathrm{E}>E_{1}$. The normalization constant A depends on the composition, and is given by:

$\mathfrak{G}_{5}=\frac{N !}{\prod_{i=1}^{5} N_{i}}=A \frac{\Delta E^{N+1}}{N+1}$

where $\mathfrak{c}_{5}$ is the number of configurations (sites occupancies) in the iso-composition alloy with fixed mole numbers $\left\{N_{i}\right\}$ and $N=\sum_{i=1}^{5} N_{i}$ The partition function is thus $\mathrm{Z}=\mathrm{A} \Gamma_{N}$ with $\Gamma_{N}=\int_{E_{0}}^{E_{1}}\left(E-E_{0}\right)^{N} e^{-\beta E} d E$.

$\Gamma_{N}$ fulfills the recurrence relation:

$\Gamma_{N}=\mathrm{H}_{N}+\frac{N}{\beta} \Gamma_{N-1}$

with $\mathrm{H}_{N}=\frac{\mathrm{e}^{-\beta E_{1}}}{-\beta} \Delta E^{N}$ and $\Gamma_{0}=\frac{e^{-\beta E_{1}}-e^{-\beta E_{0}}}{-\beta}=\mathrm{H}_{0}-\frac{e^{-\beta E_{0}}}{-\beta}$. 
from which it follows that:

$\Gamma_{N}=\sum_{k=0}^{N} \frac{\mathrm{N} !}{(N-k) !} \frac{1}{\beta^{k}} \mathrm{H}_{N-k}+\frac{N !}{\beta^{N+1}} e^{-\beta E_{0}}$

In practice, $\Delta E \gg 1 / \beta$, which implies that $\mathrm{H}_{N-k}=\frac{e^{-\beta E_{0}}}{-\beta} e^{-\beta \Delta E} \Delta E^{N-k} \rightarrow 0, \forall N-k$. Hence:

$\Gamma_{N} \cong \frac{N !}{\beta^{N+1}} e^{-\beta E_{0}}$
The expression for the alloy free energy follows immediately:

$$
F=-\frac{1}{\beta} \ln Z=\mathrm{E}_{0}-\frac{1}{\beta} \ln \mathfrak{E}_{5}+\frac{1}{\beta} \ln \frac{(\beta \Delta E)^{N+1}}{(N+1) !}
$$

In this expression, the quantities $E_{0}, \mathfrak{C}_{5}$ and $\Delta E$ are functions of $\left\{N_{i}\right\}$. Moreover, $E_{O}$ and $\Delta E$ are supposed to be extensive: $\Delta E\left(\left\{N_{i}\right\}\right)=N \Delta e\left(\left\{x_{i \neq i_{0}}\right\}\right)$ where $\Delta e=\frac{\Delta E}{N}$ is the energy dispersion per atom, and similarly $E_{0}\left(\left\{N_{i}\right\}\right)=N e_{0}\left(\left\{x_{i \neq i_{0}}\right\}\right)$. Application of the gen-

$$
\alpha_{i j}=\mathbf{0}
$$

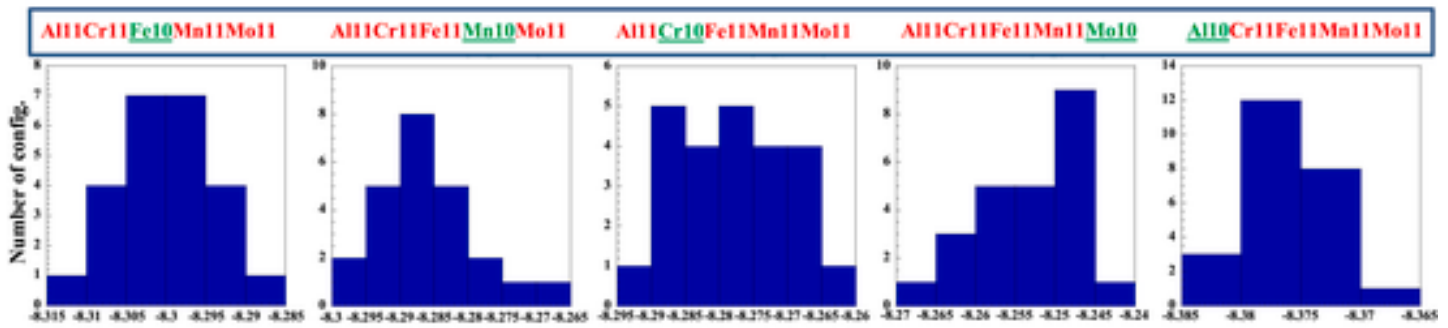

(a) Opt1N

Energy (eV)

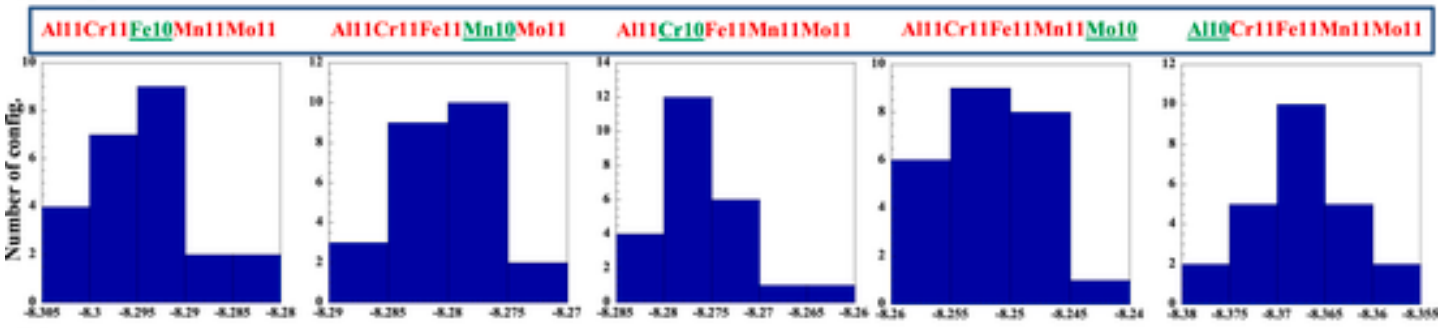

(b)Opt1N2N

Energy $(\mathrm{eV})$

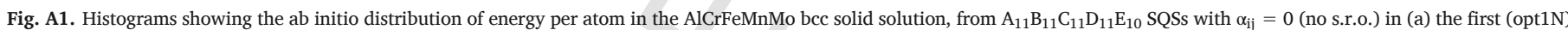

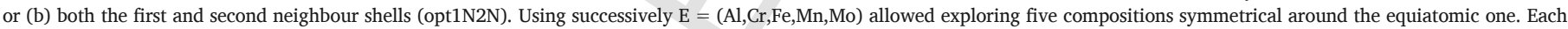
histogram is drawn from a panel of 24 structures obtained via the full set of (A,B,C,D) permutations. The width of a bin is $5 \mathrm{meV}$.

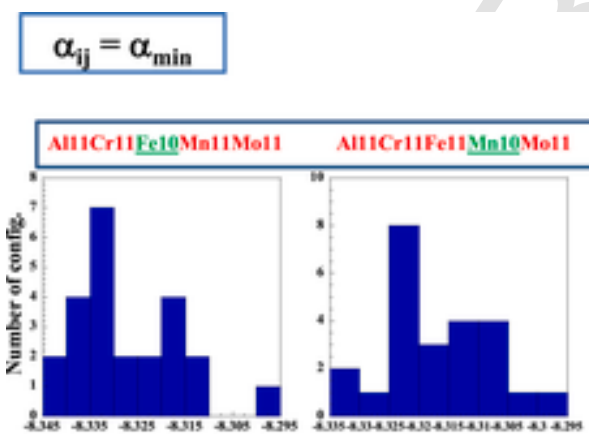

(a) Opt1N

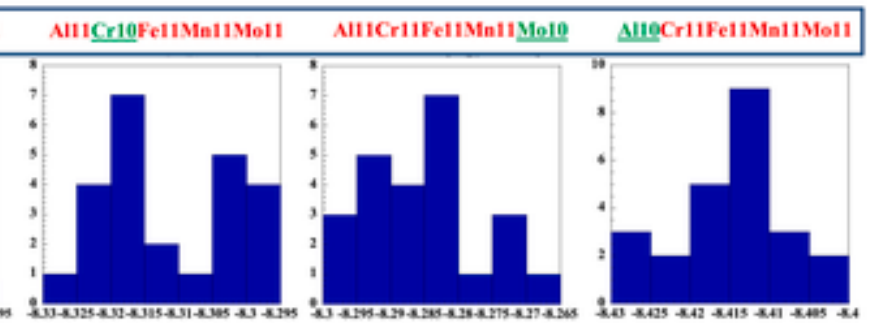

Energy (eV)

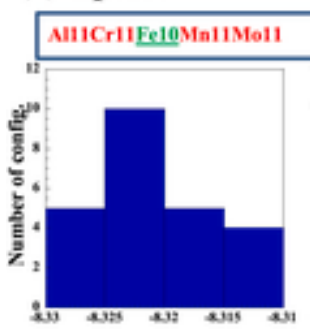

(b)Opt1N2N

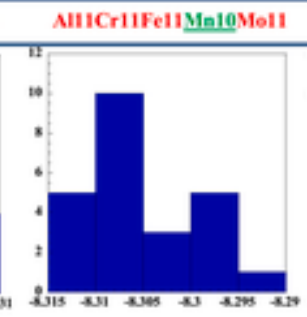

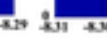

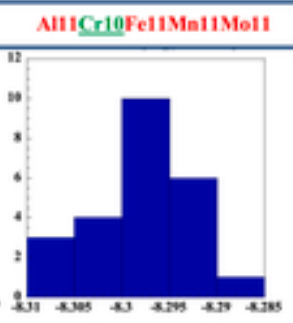

Energy (eV)

Fig. A2. Same as Fig. A1, for the case $\alpha_{i j}=\alpha_{\min }$. 


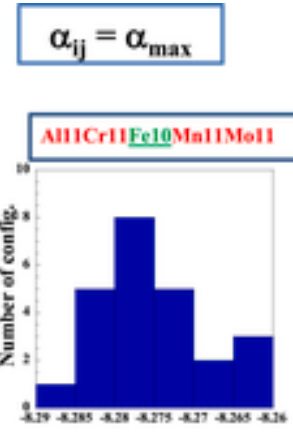

(a) Opt1N

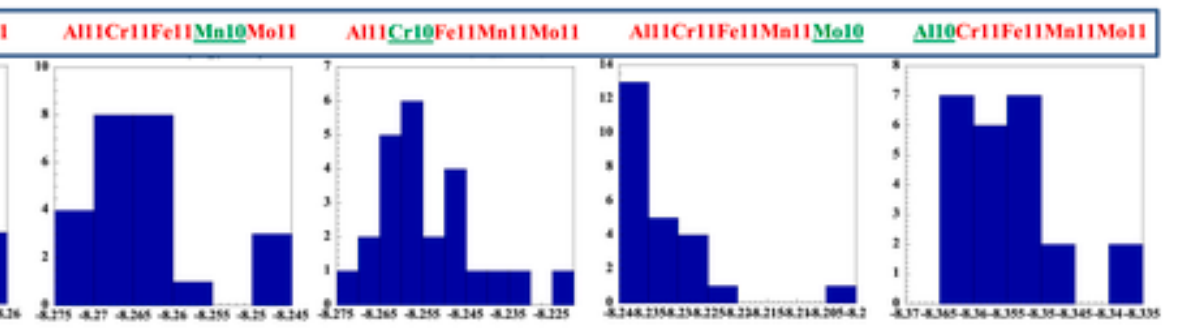

Energy (eV)

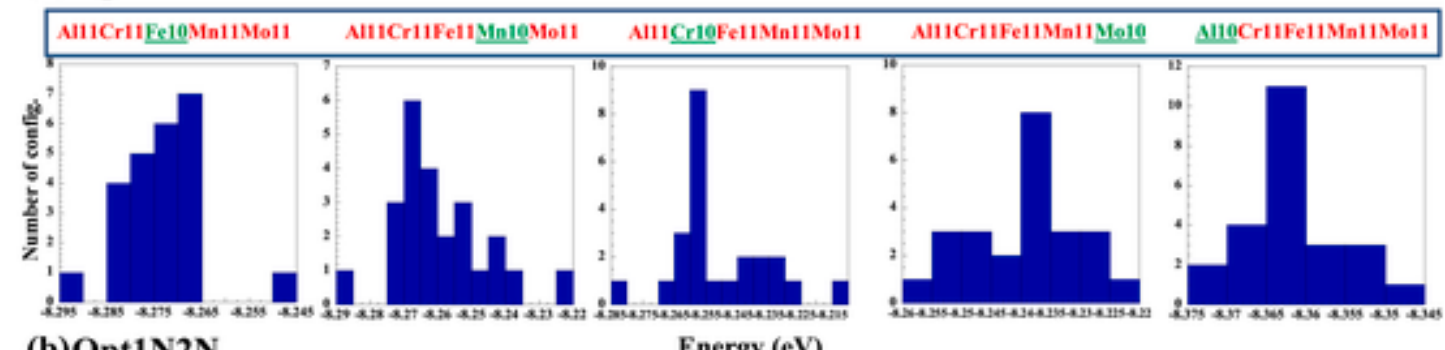

(b)Opt1N2N

Energy (eV)

Fig. A3. Same as Fig. A1, for the case $\alpha_{i j}=\alpha_{\max }$.
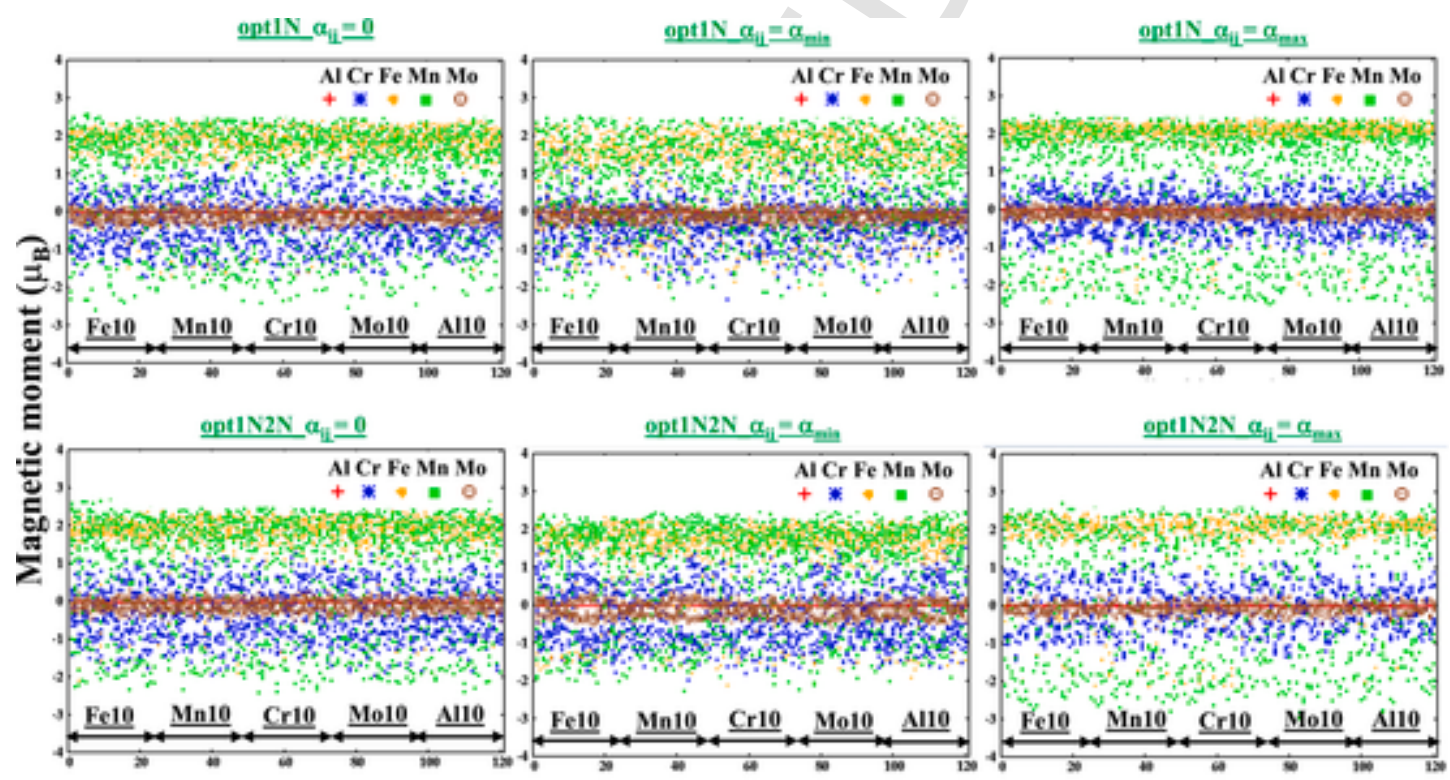

Permutation index

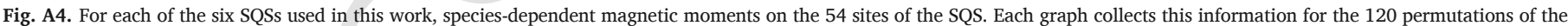
SQS considered. On each graph, the permutation index is directly related to the nearly equiatomic composition (24-element permutation panel for each composition coordinate).

eral relation $\mu_{i}=\left(\frac{\partial F}{\partial N_{i}}\right)_{T, V, N_{j \neq i}}$ straightforwardly yields the chemical potentials, Eqs. (6) and (7).

Appendix 2. : Energy histograms for each SQS

See Appendix Figs. A1-A3.

Appendix 3. : Magnetic moments for each SQS

See Appendix Fig. A4.

\section{References}

[1] D.B. Miracle, O.N. Senkov, A critical review of high entropy alloys and related concepts, Acta Mater. 122 (2017) 448-511.

[2] O.N. Senkov, J.D. Miller, D.B. Miracle, C. Woodward, Accelerated exploration of multi-principal element alloys for structural applications, Calphad 50 (2015) 32-48.

[3] T. Stasiak, S.N. Kumaran, M. Touzin, F. Béclin, C. Cordier, Novel multicomponent powders from the AlCrFeMnMo family synthesized by mechanical alloying, Adv. Eng. Mater. 21 (5) (2019) 1900808.

[4] S.-H. Alex Zunger, L.G. Ferreira Wei, James E. Bernard, Special quasirandom structures, Phys. Rev. Lett. 65 (1990) 353-356.

[5] G. Kresse, J. Hafner, Ab initio molecular dynamics for liquid metals, Phys. Rev. B 47 (1993) 558-561.

[6] G. Kresse, D. Joubert, From ultrasoft pseudopotentials to the projector augmented-wave method, Phys. Rev. B 5 (1999) 1758-1775. 
[7] J.P. Perdew, K. Burke, M. Ernzerhof, Generalized gradient approximation made simple, Phys. Rev. Lett. 77 (1996) 3865-3868.

[8] J.P. Perdew, K. Burke, M. Ernzerhof, Generalized gradient approximation made simple, Phys. Rev. Lett. 78 (1997) 1396-1396.

[9] H.J. Monkhorst, J.D. Pack, Special points for Brillouin-zone integrations, Phys. Rev. B 13 (1976) 5188-5192.

[10] R. Besson, J. Dequeker, L. Thuinet, A. Legris, Ab initio thermodynamics of complex alloys: the case of Al- and Mn-doped ferritic steels, Acta Mater. 169 (2019) 284-300.

[11] A. Fernández-Caballero, M. Fedorov, J.S. Wróbel, P.M. Mummery, D. Nguyen-Manh, Configurational entropy in multicomponent alloys: matrix formulation from ab Initio based Hamiltonian and application to the FCC Cr-Fe-Mn-Ni system, Entropy 21 (2019) 68.

[12] F. Reif, Fundamentals of statistical and thermal physics, seventeenth ed., McGraw-Hill International Book Company, 1984.

[13] R. Besson, Point defects in multicomponent ordered alloys: methodological issues and working equations, Acta Mater. 58 (2010) 379-385.

[14] D. Sobieraj, J.S. Wróbel, T. Rygier, K.J. Kurzydłowski, O. El Atwani, A. Devaraj, E. Martinez, D. Nguyen-Manh, Chemical short-range order in derivative Cr-Ta-Ti-V-W high entropy alloys from the first-principles thermodynamic study, Phys. Chem. Chem. Phys. 22 (2020) 23929-23951.

[15] M.C. Troparevsky, J.R. Morris, P.R.C. Kent, A.R. Lupini, G.M. Stocks, Criteria for predicting the formation of single-phase high-entropy alloys, Phys. Rev. X 5 (2015) 011041.

[16] L.J. Santodonato, P.K. Liaw, R.R. Unocic, H. Bei, J.R. Morris, Predictive multiphase evolution in Al-containing high-entropy alloys, Nat. Commun. 9 (2018) 4520.

[17] D. Tingaud, R. Besson, Point defect phonons in intermetallics: $\mathrm{NiAl}_{3}$ by atomic-scale simulation, Phys. Status Solidi C. 6 (2009) 2008-2011.

[18] D. Tingaud, R. Besson, Point defects and diffusion in ordered alloys: an ab initio study of the effect of vibrations, Intermetallics 45 (2014) 38-45. 